\title{
El Estrés y Bruxismo por COVID-19 como Factores de Riesgo en la Enfermedad Periodontal
}

\author{
Stress and Bruxism Due to COVID-19 as Risk Factors in Periodontal Disease
}

\author{
Michelle Morón Araújo, ${ }^{1,2}$
}

MORÓN, A. M. El estrés y bruxismo por COVID-19 como factores de riesgo en la enfermedad periodontal. Int. J. Odontostomat., 15(2):309-314, 2021.

RESUMEN:Por la pandemia de COVID- 19 que inició año 2020 se ha venido desarrollando problemas de gran consecuencia afectando a la salud general, incluyendo a la salud mental y bucodental. El estrés, y angustia ha generado manifestaciones clínicas psicosociales como la ansiedad, depresión entre otros. Problemas orales como el bruxismo definido como el rechinamiento de dientes inconscientemente y parafunción mandibular persistente trae como consecuencias problemas patológicos en la articulación temporomandibular, los músculos asociados, ligamento periodontal y la fractura de dientes. El estrés siendo un factor psicosocial que aumentó por esta pandemia es un factor de riesgo para desarrollar enfermedad periodontal. Ha sido estudiado una persona con estrés libera moléculas en el organismo que llevan a depresión, y este metabolismo puede llevar a un desánimo repercutido en poca disposición para realizar los hábitos de higiene oral o buscar en el tabaco para liberar el estrés, por eso estas son condiciones que van en círculo vicioso que afectan la salud periodontal. El estrés crónico es nocivo ya que se mantiene a lo largo del tiempo estimulando al hipotálamo en una serie de hormonas desencadenando liberación de glucocorticoides como el cortisol demostrado en estudios relacionado a una baja de defensas del organismo y este va hacer un factor de riesgo a desarrollar la enfermedad periodontal. Sumado a todo esto el bruxismo con lleva a una parafuncion donde se va a producir un trauma oclusal afectando el ligamento periodontal y al hueso. Se han reportado muchos casos de fracturas dentales a causa del estrés que genera el bruxismo. Existen muchos métodos para prevenir y eliminar el estrés como, actividad física, comida saludable entre otras que pueden tener un impacto positivo y ayudar en las afecciones psicosociales en esta pandemia de COVID 19.

PALABRAS CLAVE: enfermedad psicosocial, bruxismo, COVID-19, enfermedad periodontal, estrés.

\section{INTRODUCCIÓN}

En estos últimos años las pandemias que se han presentado han desafiado al ser humano que todavía sigue siendo vulnerable a las enfermedades infectocontagiosas. En Diciembre del año 2019 en la ciudad de Wuhan, provincia de Hubei en China, se presentó una serie de pacientes con síndrome respiratorio agudo. La mayoría de ellos estaban relacionados con haber trabajado o frecuentando en el mercado de pescado de Wuhan. En este mercado se vende carne de animales salvajes, incluyendo murciélagos (World Health Organization, 2020).

Esta enfermedad nueva que se propagó a muchos países de todo el mundo en los últimos meses por la pandemia de COVID-19 ha obligado al mundo a una crisis sanitaria, social, psicológica y económica. Las implicaciones del aislamiento social van de problemas físicos y mentales (World Health Organization).

Los países tuvieron que prepararse y reinventarse para responder a diferentes escenarios de salud pública, reconociendo que no existe un enfoque único para el manejo de casos y brotes de COVID-19. Cada país evaluó su riesgo, las medidas establecidas y su aceptabilidad social, e implementar rápidamente las intervenciones necesarias en la escala adecuada para detener la transmisión de COVID-19 y minimizar los impactos económicos, públicos y sociales (World Health Organization).

${ }^{1}$ Odontóloga, Pontificia Universidad Javeriana, Bogotá, Colombia.

${ }^{2}$ Periodoncista, Pontificia Universidad Católica, Buenos Aires, Argentina. 
Este virus trajo consecuencias negativas por la rápida propagación, cuarentenas obligatorias, cierre de lugares de trabajo, disminución de ingresos económicos etc. El estrés, la ansiedad e impactos psicológico y en consecuenciade esto el bruxismo como factores de riesgos desencadenantes de desarrollar enfermedades periodontales que pueden afectar significativamente nuestra salud oral y sistema estomatognatico (Ozamiz-Etxebarria et al., 2020).

Por esta situación, se desarrollaron propuestas y estrategias para la prevención y el tratamiento de los efectos psicológicos que puede crear una pandemia como la del COVID-19. Las entidades con perfiles socio-sanitarios, administrativos y educativos, como las federaciones y academias han diseñado planes y programas para ayudar a combatir el estrés, como en Pekín (China). En la universidad de Pekín desarrollaron manuales de salud mental para instruir, motivar y enfrentarse al estrés y problemas psicológicos que trajo esta pandemia de COVID-19 (Ozamiz-Etxebarria et al., 2020).

Estos modelos de planes preventivos y proyectos para la salud mental de la población se están promoviendo en diferentes países incluyendo latinoamericanos. El bruxismo como consecuencia del estrés y ansiedad es un hábito involuntario con problemas funcionales del sistema estomatognatico que consiste en el rechinamiento de los dientes que en la mayoría de las veces se realiza inconscientemente cuando las personas duermen de noche (Sutthiboonyapan \& Wang, 2019).

El bruxismo puede desarrollar afecciones en los nervios de los músculos de la cabeza, cuello, hombros que se enlazan hasta la articulación temporomandibular (ATM), y la mandíbula con el cráneo. De esta manera, las sobrecargas musculares pueden producir problemas de bruxismo. (Sutthiboonyapan, Wang 2019).

Los problemas relacionados con el sueño pueden tener una incidencia directa sobre el apretamiento involuntario de los dientes. Debido a la situación actual que estamos viviendo muchos pacientes han tenido dificultades para conciliar el sueño. En este sentido, esa tensión puede pasar a los dientes y a la mandíbula. Esto a su vez, puede empeorar los problemas de bruxismo (Sutthiboonyapan \& Wang).

Una discrepancia oclusal y una parafuncion puede producir el desarrollo de la periodontitis por trauma oclusal que se define como una lesión en los tejidos de soporte del diente o del implante, es el resultado de hábitos parafuncionales como el bruxismo. Se ha demostrado que acelera la enfermedad periodontal y también contribuye a las complicaciones del implante, lo que afecta negativamente al éxito a largo plazo de la terapia periodontal e implantológico.

El estrés y la relación con la enfermedad periodontal: El estrés que se ha desencadenado por la pandemia del COVID 19 ha conducido a desarrollar muchos problemas de salud general incluyendo, ansiedad, depresión, problemas mentales, entre otros. El estrés puede desencadenar problemas bucales como el bruxismo, trastornos temporomandibulares, fracturas dentales y la enfermedad periodontal.

El COVID-19 puede inducir la activación de mastocitos, estrés psicológico, tormenta de citocinas, neuroinflamación, enfermedades inflamatorias sistémicas, enfermedades neuroinflamatorias, traumatismo craneoencefálico accidente cerebrovascular y trastornos por estrés (Kempuraj et al., 2020).

De acuerdo a la bibliografía consultada se describe el impacto del estrés en la enfermedad periodontal, la evidencia científica del estrés psicológico como factor de riesgo de periodontitis es fragmentaria y se ha basado principalmente en estudios basados en cuestionarios o biomarcadores (Haririan et al., 2018).

Hay indicios que las neuronas pueden secretar citocinas y quimiocinas proinflamatorias que empeoran las reacciones inflamatorias crónicas en el periodonto y comprometen la capacidad inmunológica. Los estudios in vitro muestran que los niveles altos de cortisol pueden contribuir al aumento del crecimiento de Porphyromonas gingivales (Haririan et al.).

La enfermedad periodontal tiene un vínculo con la placa bacteriana y los malos hábitos e uso inadecuado de los elementos de higiene oral. Actualmente esta relación produce una alteración inflamatoria que se ha relacionado con las enfermedades sistémicas (Jiménez-Martínez et al., 2013; Sánchez-Villamil et al., 2020).

El estrés oxidativo está estrechamente vinculado con la enfermedad periodontal por poseer una relación con los niveles endógenos antioxidantes que 
poseen numerosos radical libre como enzimas oxidantes como el superóxido dismutasa y glutatión peroxidasa (Jiménez-Martínez et al.; Sánchez-Villamil et al.).

Los estudios conllevan a relacionar la enfermedad periodontal inflamatoria con los factores etiológicos generales que provocan la ruptura de sistemas fisiológicos de inhibición de la peroxidación lipídica, realizan un bajo nivel de protección antioxidante de los tejidos periodontales. Esta conexión con los factores locales conducen a la migración de neutrófilos hacia la gingiva y el fluido gingival (Sánchez-Villamil et al.).

La aceleración de los leucocitos en fagocitosis, produce la liberación de especies reactivas del oxígeno, que conducen al desencadenamiento de la peroxidación lipídica de los tejidos periodontales y a la activación de proteasas. La peroxidación lipídica constituye el mecanismo que desencadena el desarrollo de cambios morfo funcionales en el periodonto y sus vasos, lo cual resulta en destrucción del colágeno y reabsorción ósea (García Montes de Oca, 2004; Chen et al., 2020).

En un estudio se realizó evaluación de las profundidad de las bolsas al sondaje moderada (4-6 mm) y un nivel de inserción moderado $(4-6 \mathrm{~mm}$ ) presentaba una asociación positiva con unos resultados en los cuestionarios de valoración del estrés y la ansiedad, lo cual ha llevado a investigar y concluir sobre aquellas personas con unos niveles más altos de estrés y de ansiedad son más propensas a desarrollar enfermedad periodontal (García Montes de Oca).

Se concluye por los autores que los microrganismos patógenos son el factor principal de la enfermedad periodontal, Controlar o mantener el estrés negativo será clave para disminuir la predisposición que un individuo tenga para desarrollar patologías gingivales y periodontales (García Montes de Oca; Chen et al.).

Bruxismo por estrés y ansiedad relación con la enfermedad periodontal: Ha sido bien documentado el fuerte impacto que COVID-19 está teniendo en los problemas psicológicos, donde una parte significativa de la población ha informado de ansiedad moderada a severa. Los trabajadores de la salud, las amas de casa, principalmente mujeres, también se enfrentan a un aumento de los niveles de ansiedad y estrés (Almeida-Leite et al., 2020).
Los factores psicosociales en el desarrollo y mantenimiento de los trastornos temporomandibulares (TMD) y la alta prevalencia de trastornos psicológicos en pacientes con TMD, principalmente en aquellos que padecen trastornos de los músculos masticatorios. Además, existe una relación significativa entre desordenes temporomandibulares doloroso, depresión y ansiedad (Horesh \& Brown, 2020).

El estrés se define como un estado o proceso psíquico, químico y/o emocional que produce tensión lo cual conlleva a un desarrollo patologíco psíquico y en la salud general. Se han realizado estudios donde analizan el estrés con el bruxismo lo cual se ha evidenciado la vulnerabilidad y el nivel del estrés en pacientes que presentan bruxismo (Herrero et al., 2019).

El estrés, la ansiedad y la depresión son factores de riesgo muy comunes y que influyen como vías de desarrollar el bruxismo, los desórdenes del sueño, tales como apnea del sueño, síndrome de movimiento de las extremidades y ronquidos, representan factores de riesgo comúnmente asociados con el bruxismo.

El bruxismo es el hábito de apretar o rechinar los dientes excesivamente. Este trastorno puede afectar a adultos y a niños, y puede ocurrir de día o de noche. Algunas personas no tienen síntomas, pero otras pueden experimentar dolores de cabeza, mandibulares, articulares, dentales etc. (Ordoñez-Plaza et al., 2016).

Los pacientes con altos niveles de estrés tienen casi 6 veces más probabilidades de reportar bruxismo. La contracción sostenida de los músculos de la cabeza y el cuello también está relacionada con una postura corporal requerida asociada a la respuesta de lucha o huida. Por lo tanto, la contracción muscular en el bruxismo despierto podría ser parte del comportamiento de defensa asociado con la ansiedad y el estrés (Qu \& Zhou, 2020).

Las enfermedades psicosomáticas orales estrechamente relacionadas con el estado psicológico del paciente, como la enfermedad de la mucosa oral, la enfermedad de la articulación temporomandibular, el bruxismo, la enfermedad periodontal, etc. Se cree que los pacientes con estos trastornos podrían ser más susceptibles al impacto de los eventos de estrés y necesitarían más atención por parte de los odontólogos (Fig. 1). 


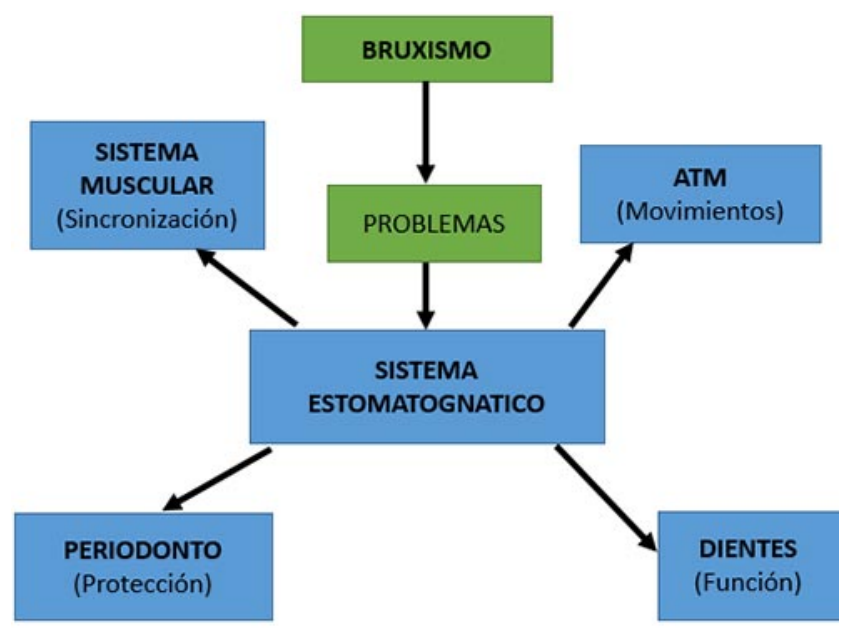

Fig 1. Afecciones producidas por bruxismo.

El trauma oclusal es el daño que produce cambios en los tejidos del aparato de inserción como resultado de las fuerzas oclusales, puede estar causado por inestabilidad oclusal (discrepancias oclusales, migraciones dentales), hábitos parafuncionales como bruxismo o ambas. Es el fracaso de la estructura de soporte para resistir o adaptarse a estas fuerzas (Gélvez Vera et al., 2016).

\section{Existen dos tipos de trauma oclusal:}

1.Primario: lesión del aparato de inserción, provocada por fuerzas oclusales excesivas sobre un diente o dientes con soporte periodontal normal.

2.Secundario: lesión en el aparato de inserción, provocada por fuerzas oclusales normales o excesivas aplicadas sobre un diente o dientes con soporte periodontal reducido.

En ambos casos, estas lesiones corresponden a cambios que se pueden evidenciar por alteraciones en el hueso de soporte y en el ligamento periodontal, este ligamento se ensancha a expensas de la reabsorción ósea, se presentan defectos óseos angulares, hay movilidad dental, pero aun así no necesariamente va a presentar bolsas periodontales, a no ser que se combine una lesión inflamatoria de origen bacteriano preestablecida (Carranza, 1998).

Esta pandemia por Covid-19 ha demostrado que diversos tipos de síntomas neuropsiquiátricos, como encefalopatía, cambios de humor, psicosis, disfunción neuromuscular y procesos desmielinizantes esto pueden estar acompañado por una infección viral aguda y/o pueden seguir a la infección durante semanas, meses y también presentarse en pacientes recuperados (Troyer et al., 2020).

La OPS/OMS (Organización panamericana de la salud/ Organización mundial de la salud), junto con sus colaboradores, han brindando orientación y asistencia a las regiones durante la pandemia COVID-19, para ayudar a apoyar el bienestar mental y general de todas las personas, especialmente de aquellos que podrían necesitar apoyo adicional durante estos difíciles momentos. En su página se encuentran guías y recomendaciones donde se brindan orientaciones y consejos para manejar la ansiedad el estrés y las afecciones psicológicas que ha traído esta pandemia.

Es importante realizar una consulta odontológica cuando hay una presencia de bruxismo y cuando se presenten síntomas dolorosos, en la articulación temporomandibular, fractura dental, desgastes, movilidad dental entre otros. Se pueden realizar tratamientos como las férulas oclusales, ajustes oclusales, y/o ferulizacion (Sutthiboonyapan \& Wang).

\section{CONCLUSIONES}

Esta pandemia por COVID-19 ha sido de gran impacto tanto para la salud física como para la salud mental y el bienestar de sociedades que se han visto afectadas gravemente por esta crisis. El cambio de vida a causa de esta pandemia ha generado consecuencias socioeconómicas, el miedo al virus y su propagación, así como las preocupaciones asociadas, tienen un fuerte impacto en la salud mental de la población mundial.

El bruxismo generado por ansiedad y estrés han sido un problemas en la salud oral en esta pandemia y se ha manifestado como en los desgastes y fracturas dentales, movilidad, afecciones en el ligamento periodontal entre otras. Muchos odontólogos han visto en sus consultas más dientes rotos de lo normal a causa de estos problemas psicosociales que ha generado esta pandemia por COVID-19.

Las placas oclusales y/o miorrelajantes ayudan a los dientes a no tener un desgaste y fractura por bruxismo estos elementos ayudan al ligamento periodontal y al hueso a no presentar una injuria.

Está comprobado y con ayuda de la organización mundial de la salud establece pautas para disminuir el estrés como: 
- Mantenerse informado.

- Seguir las rutinas de higiene general de lavado de manos y en esta incluida el cepillado e higiene oral, ejercicio, descanso, comida saludable.

- Meditar y orar.

- Reducir la exposición de noticias negativas.

- Contacto social ya sea por teléfono, virtual, redes sociales entre otras.

- Evitar el consumo de drogas y alcohol.

- Controlar el tiempo de pantalla.

- No abusar de video juegos.

- Utilizar adecuadamente las redes sociales.

- Ayudar a los demás.

- Apoyar al personal sanitario.

MORÓN, A. M. Stress and bruxism due to COVID-19 as risk factors in periodontal disease. Int. J. Odontostomat., 15(2):309-314,2021.

ABSTRACT: Due to the COVID-19 pandemic of this year 2020, problems of great consequence have been developing in consequences in general health, including mental and oral health. Stress and anguish have generated psychosocial clinical manifestations such as anxiety, depression, among others. Oral problems such as bruxism defined as unconscious grinding of teeth and persistent mandibular parafunction results in pathological problems in the temporomandibular joint, associated muscles, periodontal ligament and tooth fracture. Stress, being a psychosocial factor that increased by this pandemic, is a risk factor for developing periodontal disease. A person with stress has been studied to release molecules in the body that lead to depression, and this metabolism can lead to discouragement, impaired in a reluctance to perform oral hygiene habits or to look at tobacco to release stress, so these are conditions that go vicious circle that affect periodontal health. Chronic stress is harmful since it is maintained over time by stimulating the hypothalamus in a series of hormones, triggering the release of glucocorticoids such as cortisol, shown in studies related to a decrease in the body's defenses and this will become a risk factor to develop periodontal disease. In addition to all this, bruxism leads to a parafunction where an occlusal trauma will occur affecting the periodontal ligament and the bone. Many cases of dental fractures have been reported due to the stress generated by bruxism. There are many methods to prevent and eliminate stress such as physical activity, healthy food among others that can have a positive impact and help in psychosocial conditions in this COVID 19 pandemic.

KEY WORDS: psychosocial illness, bruxism, COVID-19, periodontal disease, stress.

\section{REFERENCIAS BIBLIOGRÁFICAS}

Almeida-Leite, C. M.; Stuginsk-Barbosa, J. \& Rodrigues Conti, P. C. How psychosocial and economic impacts of COVID-19 pandemic can interfere on bruxism and temporomandibular disorders? J. Appl. Oral Sci., 28:e20200263, 2020.

Carranza, F. Periodontologia Clínica de Glickman. Ciudad de México, McGraw-Hill-Interamericana,1998.

Chen, M.; Cai, W.; Zhao, S.; Shi, L.; Chen, Y.; Li, X.; Sun, X.; Mao, Y.; He, B.; Hou, Y.; et al.,. Oxidative stress-related biomarkers in saliva and gingival crevicular fluid associated with chronic periodontitis: A systematic review and metaanalysis. J. Clin. Periodontol., 46(6):608-22, 2019.

García Montes de Oca, A. L. Influencia del estrés oxidativo en la enfermedad periodontal. Rev. Cienc. Med. La Habana, 10(2), 2004. Disponible en: http://revcmhabana.sld.cu/index.php/ $\mathrm{rcmh} /$ article/view/141

Gélvez Vera, M. A.; Velosa Porras, J. \& Pérez Gutiérrez, B. Efecto de las fuerzas oclusales sobre el periodonto analizado por elementos finitos. Univ. Odontol., 35(74):85-92, 2016.

Haririan, H.; Andrukhov, O.; Böttcher, M.; Pablik, E.; Wimmer, G.; Moritz, A. \& Rausch-Fan, X. Salivary neuropeptides, stress, and periodontitis. J. Periodontol., 89(1):9-18, 2018.

Herrero, S. Y.; Arias, M. Y. \& Cabrera, H. Y. Vulnerabilidad y nivel de estrés en pacientes con bruxismo. Rev. Cuba. Estomatol., 56(3), 2019. Disponible en: https://www.medigraphic.com/cgi$\mathrm{bin} / \mathrm{new} /$ resumen.cgi?IDARTICULO=93724

Horesh, D. \& Brown, A. D. Traumatic stress in the age of COVID19: A call to close critical gaps and adapt to new realities. Psychol. Trauma, 12(4):331-5, 2020.

Jiménez-Martínez, R.; Mendieta, Z. H.; Scougall-Vilchis, R. J.; Colín, F. M. C. \& Romero, F. M. S. Estrés oxidativo, antioxidantes y enfermedad periodontal. Revisión bibliográfica. Rev. ADM, 70(6):298-301, 2013.

Kempuraj, D.; Selvakumar, G. P.; Ahmed, M. E.; Raikwar, S. P.; Thangavel, R.; Khan, A.; Zaheer, S. A.; Iyer, S. S.; Burton, C.; James, D.; et al., COVID-19, mast cells, cytokine storm, psychological stress, and neuroinflammation. Neuroscientist, 26(5-6):402-14, 2020.

Ordoñez-Plaza, M. P.; Villavicencio-Caparó, E.; AlvaradoJiménez, O. R. \& Vanegas-Avecillas,M. E. Prevalencia de bruxismo de vigilia evaluado por auto-reporte en relación con estrés, ansiedad y depresión. Rev. Estomatol. Herediana, 26(3):147-55, 2016.

Ozamiz-Etxebarria, N.; Dosil-Santamaria, M.; PicazaGorrochategui, M. \& Idoiaga-Mondragon, N. Stress, anxiety, and depression levels in the initial stage of the COVID-19 outbreak in a population sample in the northern Spain. Cad. Saude Publica, 36(4):e00054020, 2020.

Qu, X. \& Zhou, X. D. Psychological intervention for patients with oral disease during the pandemic period of COVID-19. Zhonghua Kou Qiang Yi Xue Za Zhi, 55(4):235-40, 2020.

Sánchez-Villamil, J. P.; Pino-Vélez, C.; Trejos-Suárez, J.; Cardona, N.; España, A. L. \& Alfonso, P. A. Marcadores salivales de estrés oxidativo y agentes patógenos periodontales en pacientes con periodontitis en Santander, Colombia. Biomédica, 40(Supl. 1):113-24, 2020. Disponible en: https:// revistabiomedica.org/index.php/biomedica/article/view/5149

Sutthiboonyapan, P. \& Wang, H. L. Occlusal splints and periodontal/implant therapy. J. Int. Acad. Periodontol., 21(1):45-50, 2019. 
MORÓN, A. M. El estrés y bruxismo por COVID-19 como factores de riesgo en la enfermedad periodontal. Int. J. Odontostomat., 15(2):309-314, 2021.

Troyer, E. A.; Kohn, J. N. \& Hong, S. Are we facing a crashing wave of neuropsychiatric sequelae of COVID-19? Neuropsychiatric symptoms and potential immunologic mechanisms. Brain Behav. Immun., 87:34-9, 2020.

World Health Organization (WHO). WHO Announces COVID-19 Outbreak a Pandemic. Copenhagen, World Health Organization, 2020. Available from: http://www.euro.who.int/ en/health-topics/health-emergencies/coronavirus-covid-19/ news/news/2020/3/who-announces-covid-19-outbreak-apandemic
Dirección para correspondencia:

Michelle Morón Araújo

Odontóloga ,Pontificia Universidad Javeriana

Bogotá - COLOMBIA

Periodoncista Pontificia Universidad Católica

Buenos Aires - ARGENTINA

E-mail: michellemoronaraujo@gmail.com 